\title{
High-Frequency Mechanical Excitation of a Silicon Nanostring with Piezoelectric Aluminum Nitride Layers
}

\author{
Alessandro Pitanti®, ${ }^{1, *}$ Tapani Makkonen, ${ }^{2}$ Martin F. Colombano, ${ }^{3,4}$ Simone Zanotto, ${ }^{1}$ \\ Leonardo Vicarelli®, ${ }^{1}$ Marco Cecchini, ${ }^{1}$ Amadeu Griol, ${ }^{5}$ Daniel Navarro-Urrios, ${ }^{6}$ \\ Clivia Sotomayor-Torres, ${ }^{3,7}$ Alejandro Martinez $\odot, 5$ and Jouni Ahopelto ${ }^{2}$ \\ ${ }^{1}$ NEST Laboratory, CNR - Istituto di Nanoscienze and Scuola Normale Superiore, Piazza San Silvestro 12, \\ Pisa 56217, Italy \\ ${ }^{2}$ VTT Technical Research Centre of Finland Ltd, P.O. Box 1000, Espoo, VTT FI-02044, Finland \\ ${ }^{3}$ Catalan Institute of Nanoscience and Nanotechnology (ICN2), CSIC and BIST, Campus UAB, Bellaterra, \\ Barcelona 08193, Spain \\ ${ }^{4}$ Departamento Física, Universidad Autònoma de Barcelona, Bellaterra, Barcelona 08193, Spain \\ ${ }^{5}$ Nanophotonics Technology Center, Universitat Politècnica de València, Spain \\ ${ }^{6}$ MIND-IN2UB, Departament d'Electrnica, Facultat de Fisicas, Universitat de Barcelona, Martí i Franquès, 1, \\ Barcelona 08028, Spain \\ ${ }^{7}$ Catalan Institute for Research and Advances Studies ICREA, Barcelona, Spain
}

(Received 8 April 2020; revised 29 May 2020; accepted 15 June 2020; published 17 July 2020)

\begin{abstract}
A strong trend for quantum-based technologies and applications follows the avenue of combining different platforms to exploit their complementary technological and functional advantages. Micro and nanomechanical devices are particularly suitable for hybrid integration due to the ease of fabrication at multiscales and their pervasive coupling with electrons and photons. Here, we report on a nanomechanical technological platform where a silicon chip is combined with an aluminum nitride layer. Exploiting the AlN piezoelectricity, surface acoustic waves (SAWs) are injected in the Si layer where the material has been locally patterned and etched to form a suspended nanostring. Characterizing the nanostring vertical displacement induced by the SAW, we find an external excitation peak efficiency in excess of $500 \mathrm{pm} / \mathrm{V}$ at 1-GHz mechanical frequency. Exploiting the long-term expertise in silicon photonic and electronic devices as well as the SAW robustness and versatility, our technological platform represents a candidate for hybrid quantum systems.
\end{abstract}

DOI: 10.1103/PhysRevApplied.14.014054

\section{INTRODUCTION}

Recent years have witnessed a renaissance in the investigation of micro and nanomechanical devices, pushed by the capabilities they gain when combined to photonic and/or electronic systems. Achievements such as zepto and yoctogram mass sensing [1-3], acceleration sensing as low as $10 \mu \mathrm{g} \mathrm{Hz}^{-1 / 2}$ [4], low power, light intensity [5-7] and, more recently, polarization [8] fast modulation are just a few examples of the technology enabled by opto and electromechanical nanosystems [9]. Following an avenue devoted to fundamental physics, these mescoscopic devices have rightfully entered the realm of quantum mechanics, inaugurated by the milestone achievement of ground-state cooling of the motion in micrometric sized optomechanical [10] and electromechanical [11] devices. As textbook quantum harmonic oscillators, pervasively

\footnotetext{
*alessandro.pitanti@sns.it
}

coupled with many different quantum excitations, nanomechanical systems represent the perfect tool for interfacing different physical objects, from photons to electrons, from excitons to spins. This possibility has been manifested by coherently converting information with high efficiency, with practical demonstration, and with practical demonstration of bidirectional conversion of photons from the near-infrared range (NIR) to microwaves (MW) [12-14], from NIR to NIR [15] and from MW to MW [16]. A particularly intriguing approach is to interface NIR and MW photons, the former being suitable for long-distance telecommunication (thanks to their robustness against thermal decoherence and to low-loss optical fibers), while the latter is an ideal tool for manipulating and interrogating superconducting qubits, which operate in cryogenic environments. The main challenges of realizing this kind of device lie in the very different footprints of NIR and MW resonators, which have to be mutually coupled to the very same mechanical resonator. Moreover, NIR photons suffer 
from metal-induced Ohmic losses; on the other hand, metal superconducting circuits switch to their normal phase when illuminated with photons with thermal energy above their critical temperature, which in most superconductors lies around $1 \mathrm{~K}$. To keep NIR and MW resonators well separated, a mechanical traveling-wave approach is then usually preferred.

To this end, the ideal choice falls in using surface acoustic waves (SAWs), which can oscillate at high frequency (up to tens of $\mathrm{GHz}$ ), propagate to long (millimeters) distances, and have already been used to couple together qubits separated by $100 \mu \mathrm{m}$ [17] and pushed in the quantum limit with the generation of nonclassical mechanical Fock states [18]. The SAW is a Rayleigh wave; it does not require piezoelectricity to propagate, but it is easily generated by fabricating metallic interdigitated transducers (IDTs) on a piezoelectric material. This approach requires a simple ac bias to be fed to the IDTs rather than strong pulsed lasers, which can generate a SAW in a nonpiezoelectric material through thermally induced local deformations [19]; despite the reduced generation efficiency, the latter approach has been recently applied to SOI material for optomechanical applications [20]. Within the electrically generated SAW scenario, several material platforms have been investigated [21], starting from compact builds made of GaAs [7,22-24], AlN [25], or $\mathrm{LiNbO}_{3}$ [26]. Another possibility sees the integration of piezoelectric layers on nonpiezoelectric materials; combinations such as AlN on Si for midinfrared photonics [27] or $\mathrm{ZnO}$ on $\mathrm{Si} / \mathrm{Ge}$ [28] have been reported in the literature.

In this paper, we demonstrate a technological platform that combines the optimum optical properties of Si with a piezoelectric AlN for electrical control of SAW excitation. Despite the discontinuities at the material interface, we show injection of $1-\mathrm{GHz}$ vibrations in a nanostring with external peak efficiency of about $519 \mathrm{pm} / \mathrm{V}$. Investigation of a nanostring excitation with an approximately $100-\mathrm{MHz}$ SAW in a GaAs-based platform has been previously reported, yet with no quantification of excitation efficiency [22]. The material combination we propose is interesting for reaching lossless optomechanical-based wavelength conversion, where the already demonstrated large internal efficiencies with silicon material [15] can be backed up by large external couplings. Moreover, it can be employed for the realization of extended phononic circuits on chip, which can be interfaced with electrical or optical systems, complementing similar systems in GaAs working at $500 \mathrm{MHz}$, where high $Q$ factors have been demonstrated for wavelength-scale resonant modes [24].

\section{DEVICE FABRICATION}

The device fabrication begins with the growth of 1000 $\mathrm{nm}$ of wet oxide on a $150-\mu \mathrm{m}$ silicon wafer. On the oxide, $220 \mathrm{~nm}$ of amorphous silicon is deposited by low-pressure
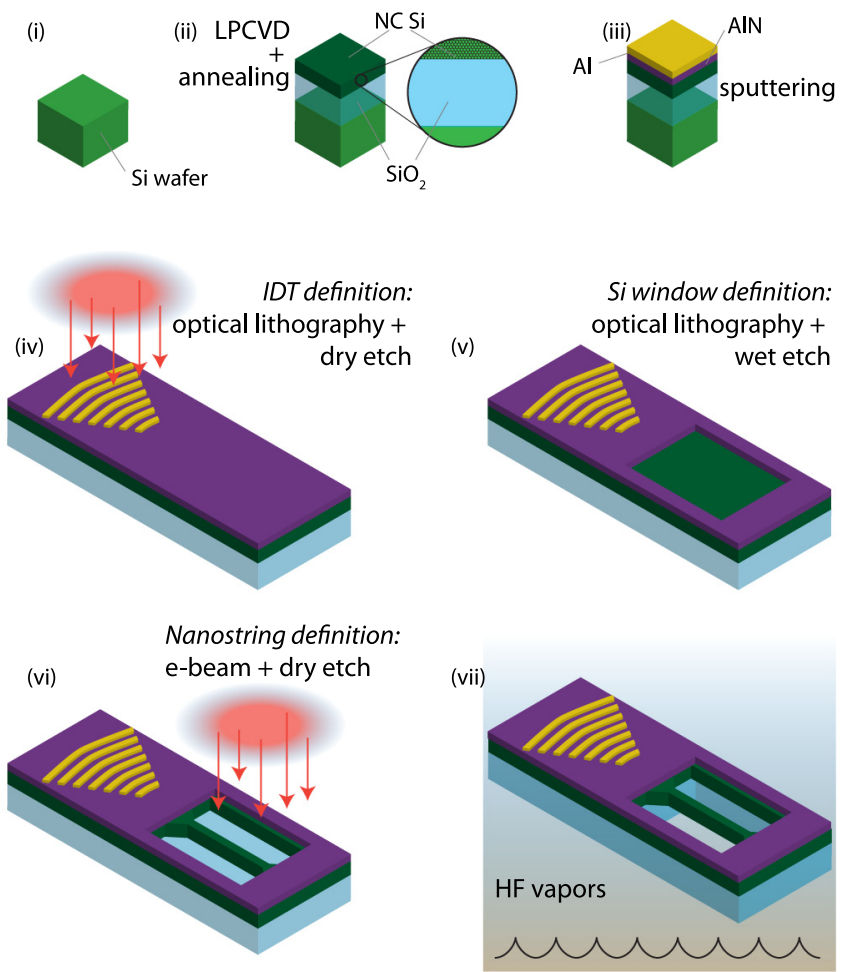

FIG. 1. Fabrication steps for material growth (i-iii) and device definition (iv-vii).

CVD at $560{ }^{\circ} \mathrm{C}$ [Fig. 1(i)]. The amorphous film is transformed to nanocrystalline by annealing at $950{ }^{\circ} \mathrm{C}$ for $60 \mathrm{~min}[29,30]$, producing a film with randomly oriented single-crystalline grains with the size distribution ranging from a few $\mathrm{nm}$ to $250 \mathrm{~nm}$ [Fig. 1(ii)]. On the annealed nanocrystalline silicon ( $\mathrm{NC} \mathrm{Si}$ ), $250 \mathrm{~nm}$ of aluminium nitride (AIN) is sputtered to form the piezoelectric actuator film [Fig. 1(iii)]. Finally, $100 \mathrm{~nm}$ of aluminium is deposited by sputtering on the AlN layer for the IDT. For operation at $1 \mathrm{GHz}$, the IDT finger periodicity is 2 $\mu \mathrm{m}$ with a $50 \%$ filling factor. Each finger in a pair is connected to different electrodes, making the system periodicity, upon the feeding of ac bias, $4 \mu \mathrm{m}$. The total number of finger pairs is 27. The IDTs are patterned by optical lithography and dry etching [Fig. 1(iv)]. The windows for the nanostrings are selectively opened in the AlN layer using slightly alkaline wet etching [Fig. 1(v)]. The nanostrings are patterned by electron-beam lithography and dry etching [Fig. 1(vi)] and, finally, the strings are released in hydrofluoric acid vapor, removing the silicon dioxide underneath the strings [Fig. 1(vii)]. The nanostring geometry has been chosen considering the typical size of silicon optomechanical nanobeams operating in the near-infrared range[30,31]; this allows an easy transfer of the obtained results to SAW-coupled optomechanical systems. In order to increase the coupling efficiency, a $2.5-\mu \mathrm{m}$-long linear tapering region is inserted at both 
ends of the nanostring. Amorphous silicon deposited by LPCVD is originally under compressive stress. During annealing, the stress changes to tensile and the amount of stress can be controlled by the annealing conditions. Having a little tensile stress in the device layer is crucial for the fabrication of released structures to avoid buckling and to control the reproducibility of mechanical properties. For the experiments, the devices are wire bonded on a PCB with coaxial connectors for the electrical input.

\section{DEVICE CHARACTERIZATION}

The mechanical action of the device has been characterized using a calibrated commercial Laser Doppler Vibrometer (LDV) from Polytech (UHF-1200). The LDV operates in a frequency range up to $1.2 \mathrm{GHz}$ and it is sensitive to mechanical out-of-plane vibrations. The 532$\mathrm{nm}$ solid-state laser is focused on the sample surface using a $100 \times$ objective, resulting in a beam spot of about $1 \mu \mathrm{m}$ in size. The full instrument operates as a standard Doppler interferometer with the added possibility of performing digital cross-correlation between the reference signal used to drive the IDT and the output signal as measured by the interferometer. This returns a measure of both quadratures, which translates in a complex time-dependent displacement $z(t)=\rho \times e^{i \omega_{m} t+i \phi}$, where $\rho$ is displacement amplitude, $\phi$ is the displacement phase, and $\omega_{m}$ is mechanical frequency. An optical microscope image of the device under test is shown in Fig. 2(a). The IDT is composed by 13 finger pairs, in the shape of circumference arcs of variable radius with the center at the first end of the silicon nanostring. This is done in order to focus the generated SAW directly at the nanostring input. The angular aperture of the arcs is $60^{\circ}$. Then device characterization begins by centering the laser spot of the interferometer roughly in the center of the IDT fed with a $1-\mathrm{GHz}$ bias. Here we perform a voltage sweep while measuring the displacement amplitude $\rho$. We observe that the device operates in the linear regime up to $2.5 \mathrm{~V}_{\text {rms }}$ and therefore we pick $2.25 \mathrm{~V}_{\mathrm{rms}}$ as the standard value for the monochromatic device characterization. A three-dimensional map of the displacement amplitude on top of the IDT is reported in Fig. 2(b). Here the color bar is in logarithmic scale with the $\mathrm{dB} \mathrm{nm}$ defined as $\rho[\mathrm{dB} \mathrm{nm}]=10 \times \log _{10}(\rho / 1[\mathrm{~nm}])$. Note that the displacement increases along $\hat{x}$, in the direction closer to the arcs' geometrical center as expected from the focusing effect of a curved IDT.

A better view of the wave periodicity on top of the IDT can be seen by inspecting the $z$ displacement in Fig. 2(c). Exploiting the common center of the IDT finger arcs, it is possible to plot the full IDT map in polar coordinates [see the sketch in Fig. 2(a)] and then integrate along the polar angle $\theta$ from $-30^{\circ}$ to $-30^{\circ}$, that is along the black lines sketched as an example in Fig. 2(c). The results are reported in Fig. 2(d), which shows a periodic signal with increasing amplitude towards the center. The Fourier transform of this signal has a narrow peak around a frequency of $1 / 4.02 \mu \mathrm{m}^{-1}$, which very well reproduces the expected periodicity of $4 \mu \mathrm{m}$. As mentioned, the signal increases towards $r=0$ due to the focusing effect of the curved IDT. More insights on the focusing effect can be gained by looking at the homogeneous region in front of the last pair of IDT fingers, highlighted in red in the optical microscope image of Fig. 3(a). A map of the displacement amplitude measured in this region is shown in Fig. 3(b).

The displacement field is spatially narrowing and increasing in amplitude when moving in $\hat{x}$ direction towards the nanostring, reaching a maximum value of
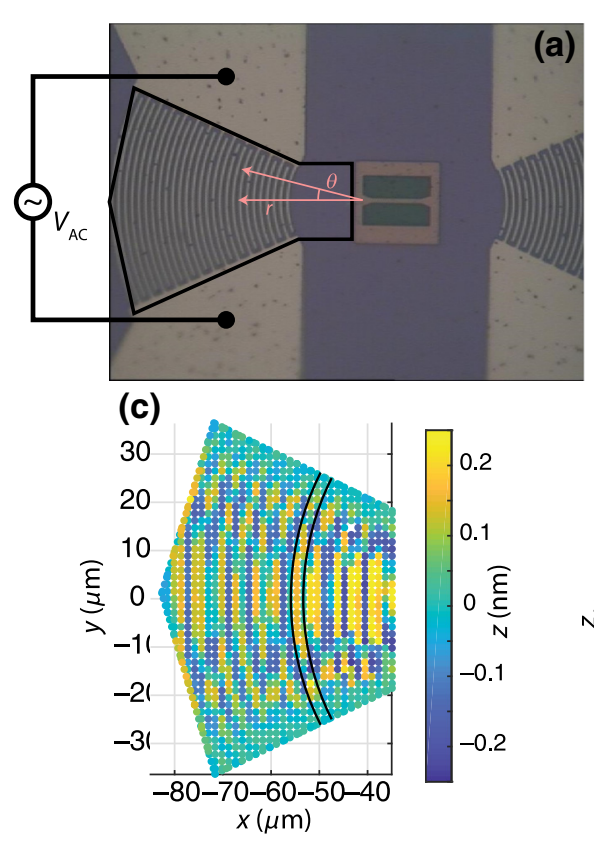

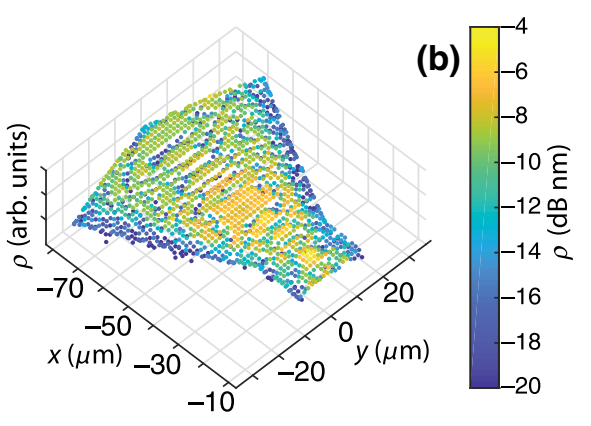

(d)

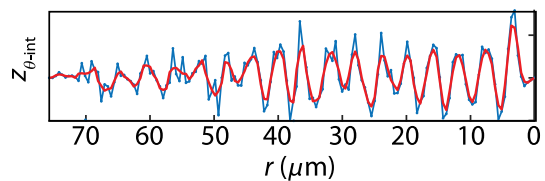

FIG. 2. (a) Microscope image of the device under test. A sketch of the measured region as well as electrical connections are added for clarity as well as the polar coordinate system (in pink). (b) Amplitude map of the selected region for an electrical signal of $2.25 \mathrm{~V}_{\mathrm{rms}}$ at $1 \mathrm{GHz}$. Note that the colorbar is in logscale. (c) Details of the complex amplitude $z$ in the outer region of the IDT. Integrating along the fingers' curvature, the displacement as a function of the geometrical center can be extracted (d), with the red line a threepoint smoothing. The increase of the amplitude as a result of the IDT focusing effect is clearly seen. 
(a)

(b)
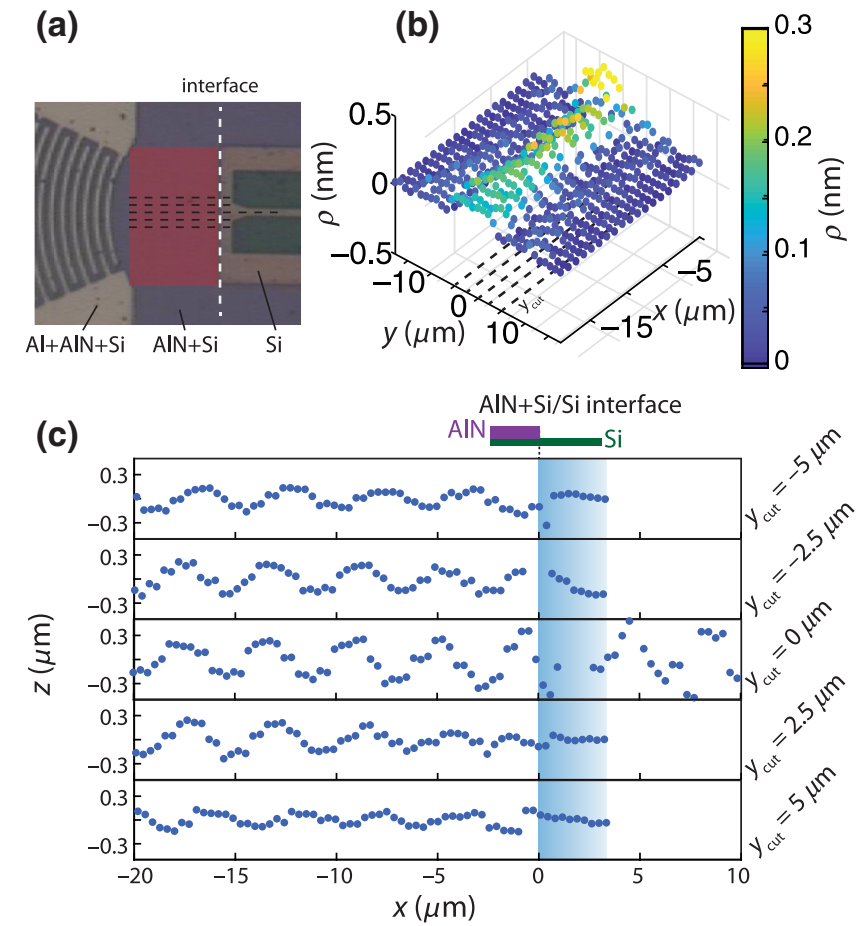

FIG. 3. (a) Microscope image of the unpatterned focusing region of the IDT. (b) Amplitude displacement map in the red region sketched in (a). (c) Complex displacement at a fixed phase for several line scans cut at fixed $y$. The interface between the silicon regions with $(\mathrm{AlN}+\mathrm{Si})$ and without $\mathrm{AlN}(\mathrm{Si})$ is further depicted. Note that the noisy signal at the interface is due to the light scattering from the almost vertical edge.

about $300 \mathrm{pm}$. This effect can be better quantified by plotting the complex displacement at a fixed phase [see Fig. 3(c)] along the dashed black lines sketched in Figs. 3(a) and 3(b). A periodic signal can be observed in every line scan with a strong difference in the signal amplitude along the central cut $(y=0)$ as compared to the other ones. In the center, the signal is increasing and strongly propagating even after the $\mathrm{AlN}+\mathrm{Si} / \mathrm{Si}$ interface. Beside the central line, the amplitude is slightly decreasing along $\hat{x}$, with a net reduction after the interface. Moreover, the absolute value of displacement is getting smaller further away from the central line. The results can be better appreciated considering the material interface, which is depicted with a blue-shaded region, while a top view of the different material stacks can be seen in Fig. 3(a). The obtained displacement translates in a focusing efficiency of $\eta_{F}=179 \mathrm{pm} / \mathrm{mW}$, where the injected power has been obtained employing the $500 \Omega$ impedance of the IDT (measurement not shown). This compares well with the focusing IDT on monolithic GaAs operating at similar frequencies $\left(\eta_{F}=33 \mathrm{pm} / \mathrm{mW}\right)$ [32] and it is of the same order of the efficiency in double-finger IDT, albeit operating at approximately $500 \mathrm{MHz}\left(\eta_{F}=228 \mathrm{pm} / \mathrm{mW}\right)$ [32]. Changing the IDT drive frequency within the excitation bandwidth produces results similar to the one extensively reported at $1 \mathrm{GHz}$ regarding the focusing effect.

The full SAW excitation bandwidth can be estimated by applying a multifrequency bias waveform at the IDT and then looking at the Fourier transform of the time signal from the LDV beam spot placed, for example, close to the focusing region [blue dot-input in Fig. 4(a)]. The displacement amplitude of the SAW excitation is reported in Fig. 4(b); the measured spectrum is a result of the superposition of three different excitation windows, each of them composed by $n=8001$ monochromatic signals, multiplexed to generate the multifrequency carrier signal. The total integrated voltage in each window is $3.5 \mathrm{~V}_{\mathrm{rms}}$; to compare the result with the one given by the monochromatic single-frequency $1-\mathrm{GHz}$ tone, one should consider that the signal is normalized considering the voltage-power spectral density (VSD), defined as $\left|V^{2}\right| /(2 \delta f)$, with $\delta f$ frequency measurement resolution (see, for example [9]). Scaling opportunely the voltage in the single-frequency measurement for a factor $\sqrt{\delta f} / n$, the approximately 300 pm displacement amplitude at $1 \mathrm{GHz}$ reported in Fig. 3 should then translate to roughly $6.5 \mathrm{pm}$ at the same frequency, given a total applied voltage $3.5 \mathrm{~V}_{\mathrm{rms}}$. Considering some uncertainty in the spatial position of the laser beam, Fig. 4(b) reproduces the expected result well, showing a displacement of $5 \pm 1 \mathrm{pm}$. The spectral shape of the signal is the one expected from a SAW excited by an IDT, whose shape can be approximated with multiple step functions in the real space. This produces a sinclike spectrum,
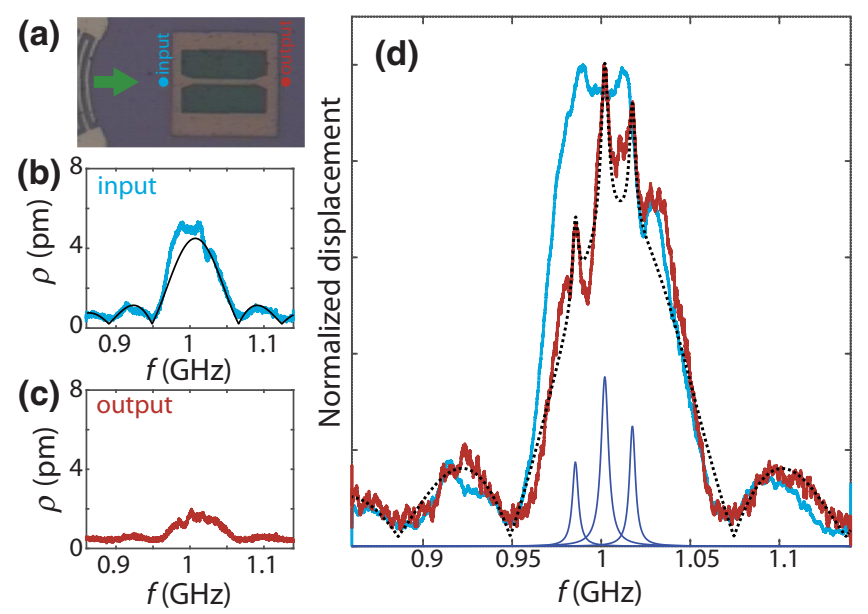

FIG. 4. (a) Optical microscope image of the nanostring devices. The arrow indicates the SAW propagation whereas the two colored dots show the acquisition position for the input (b) and output (c) displacement amplitude spectra. (d) Comparison of normalized input (blue) and output (red) displacementamplitude spectra. Best fit of the output spectrum with the IDT response function plus three Lorentzian peaks is indicated with the dashed line. The three Lorentzian peaks are also plotted individually as dark blue lines. 
with the main and secondary maxima clearly visible in the figure [33]. The spectral shape of the input signal is modified by the propagation through the mechanical nanostring; the output signal can be seen in Fig. 4(c) [red dot - output in Fig. 4(a)]. Even if proper considerations on the mechanical resonant modes should be done through spectral measurements within the nanostring itself, some technical limitations (arising from the low step-motor precision and LDV phase drifts impacting long acquisition times) forces us to perform a spectral evaluation at the device output; note that this approach is particularly useful when the device is considered a passive mechanical filter. From the reduction of the signal amplitude around the maximum of the spectrum we can extract a total loss of about $5.2 \mathrm{~dB}$. Identifying the contribution from the different loss mechanisms, including propagation loss, coupling loss, and parasitic effects due to the frame oscillations, is a challenging task in a short nanostring and will be the subject of future investigations. Normalizing the spectral displacement main peaks to one and comparing the input and output signals, it is possible to emphasize the significant difference in the main spectral features [see Fig. 4(d)]. The output spectrum can be fitted considering the sum of the IDT excitation $[\propto \operatorname{sinc}(x) / x]$ and three Lorentzian functions, centered at frequencies of $0.985,1.001$, and $1.018 \mathrm{GHz}$, respectively. These relatively sharp peaks have a low $Q$ factor of 300 , which we believe is mostly limited by the radiative loss at the nanostring ends. The peaks are likely arising from the excitation of resonant modes in the mechanical system composed by the nanostring and the released edge of the frame. Indeed, while we do not observe any strong sign of standing waves in the nanostring, which indicates high-quality-factor resonant modes, we expect that our waves have a mix of standing and traveling components. We can easily imagine that, while a strong forward wave is continuously fed by the SAW, only a weak back-scattering component can be created, due to the absence of proper mirrors in the system. Additionally, while the interface between $\mathrm{AlN}+\mathrm{Si}$ and Si regions is expected to create a strong reflection of mechanical waves, the spread mechanical wavefront can couple with the external frame with only weak waves counterpropagating within the nanostring itself. This effect complicates the evaluation of the displacement field onto the nanobeam, since the weak standing-wave component feels the full nanostring plus frame eigenstate structure. More insights on the impact of the frame can be obtained by performing extended map scans on a wide area around the nanostring region. Figure 5 reports the displacement-amplitude maps for the three resonances indicated in Fig. 4(c). There are many challenges linked to the identification of fine features in spectral maps, due to the finite size of the laser beam, vertical edge scattering, and phase drifts, which limit the maximum acquisition time in a coherent measurement; for all these reasons we consider the results in

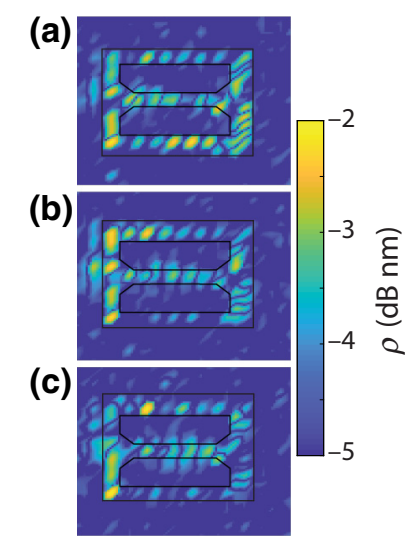

FIG. 5. Low-resolution displacement-amplitude maps (logscale) for the three resonances reported in Fig. 4, at a frequency of $0.985,1.001$, and $1.018 \mathrm{GHz}$, respectively.

Fig. 5 as a qualitative assessment of the role of the hanging frame in the vibration excitation. Despite the presence of a small phase drift, indicated by the tilted shape of the displacement-field lobes, in all cases we can observe that the frame is significantly excited with vibration amplitudes equal or even exceeding the ones of the string itself. This complicates the analysis of the system eigenmodes and could somewhat degrade the nanostring potential performance. On the other hand, we cannot exclude that the frame could be beneficial for vibration coupling into the device itself by extending the spatial profile of the mechanical eigenmode and by relaxing the strong focusing requirement for exciting the narrow silicon string.

A better assessment of the vibrations injected into the nanostring is performed by switching back to a monochromatic excitation at $2.25 \mathrm{~V}_{\text {rms }}$ and $1-\mathrm{GHz}$ frequency. By performing line scans along the nanostring length, we can estimate the displacement induced by the SAW. In addition, by performing parallel line scans on the etched region it can be checked if the SAW propagates underneath the device in the Si substrate. This is expected since the penetration depth of a SAW is of the order of the wavelength (about $4 \mu \mathrm{m}$ in our case), which is larger than the 1- $\mu$ m-thick $\mathrm{SiO}_{2}$ sacrificial layer, as shown in Fig. 6(a). A strong excitation on top of the nanostring is accompanied by weaker ones on the bottom of the device window. It is possible to verify that a coherent wave is propagating below the suspended structure by looking at the phase map in a two-dimensional scan measurement similar to the ones reported in Fig. 5. As can be seen in Fig. 6(b), a wavefront is propagating on the silicon substrate, from one end of the etched region to the other. A more quantitative assessment of the overall displacement excitation can be done by inspecting the results in Fig. 6(c), where the measured signals on the nanobeam center and on one of the two sides of the Si substrate are compared. While on the nanostring the displacement amplitude reaches about 1 


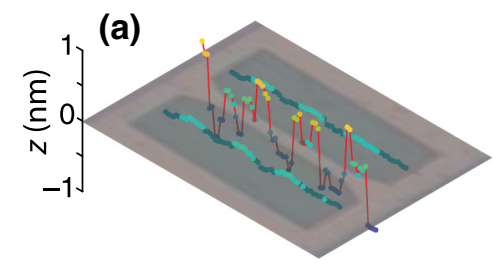

(b)

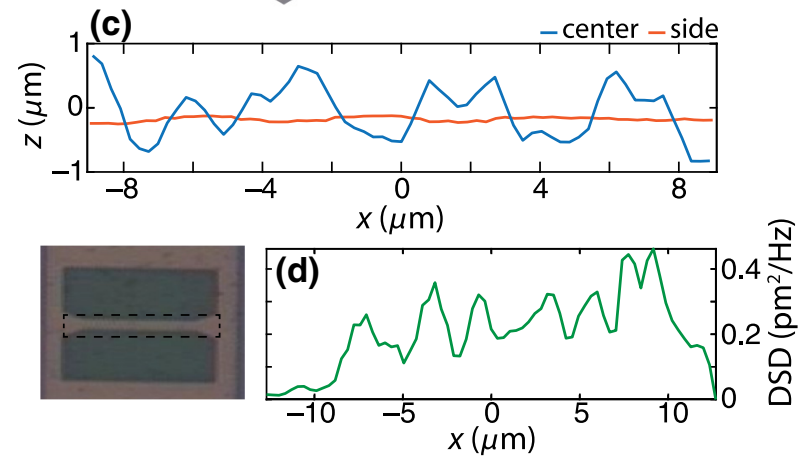

FIG. 6. (a) 3D sketch of the line-scan measurement showing a stronger excitation signal on top of the nanostring with respect to the ones coming from the residual substrate. (b) Phase map of the displacement in the etched region. (c) Direct comparison of the displacement along the central and one side line scans of (a). (d) Average DSD along the nanostring cross section.

$\mathrm{nm}$, it is reduced of about one order of magnitude on the substrate underneath. Despite the excitation splitting on the top and bottom layers, considering the average vertical displacement on the maxima and an applied voltage of 2.25 $\mathrm{V}_{\mathrm{rms}}$, we can estimate an external mechanical peak excitation efficiency, $\eta_{m}=\rho / V_{\mathrm{rms}}$, of about $519 \pm 38 \mathrm{pm} / \mathrm{V}$. The efficiency estimate is taken considering the vibrations only on the nanostring; the complicated mode structure of our systems and the limited lateral scan accuracy somewhat impact on a more refined evaluation of the excitation efficiency.

A useful quantity to evaluate is the displacement spectral density (DSD), which is defined as DSD $=|z|^{2} /(2 \delta f)$. The integral of the DSD over a mechanical resonance gives the expectation value of the displacement square, $\left\langle z^{2}\right\rangle$, even when the systems is actuated through (thermal) noisy excitation [9]. For a resonance with weak damping, it is also possible to theoretically estimate the thermal displacement square, $\left\langle z_{\mathrm{th}}^{2}\right\rangle$, using the equipartition theorem and considering a temperature $T$ [9]:

$$
\left\langle z_{\mathrm{th}}^{2}\right\rangle=\frac{k_{B} T}{m_{\mathrm{eff}} \omega_{m}^{2}},
$$

where $k_{B}$ is the Boltzmann constant, $\omega_{m}$ is resonant angular frequency, and $m_{\mathrm{eff}}$ is the effective mass of the resonator. The latter can be evaluated using commercial FEM solvers (COMSOL Multiphysics); in our case we obtain a $m_{\text {eff }}$ in a range from $2-5 \times 10^{-14} \mathrm{~kg}$, considering the few modes of the nanostring plus frame system oscillating around $\omega_{m} / 2 \pi=1 \mathrm{GHz}$. Plugging these values into Eq. (1), one finds the expected displacement square due to thermal motion at room temperature, $\left\langle z_{\mathrm{th}}^{2}\right\rangle=0.002-0.005 \mathrm{pm}^{2}$, considering the different oscillation modes as before. A direct measure of the DSD with our coherent technique can give some hints about the coherent displacement induced by the SAW. Figure 6(d) reports the average DSD obtained in a different experiment, where the area in the dashed inset has been considered. Device excitation is with a $2.25-\mathrm{V}_{\text {rms }}$ monochromatic tone oscillating at $1 \mathrm{GHz}$. DSD is plotted along the nanobeam length $(\hat{x})$, where the average is taken along the nanobeam width direction $(\hat{y})$. The measurement bandwidth is $\delta f=625 \mathrm{kHz}$. Considering a single total DSD value obtained by averaging also along $\hat{x}$, it is possible to estimate a SAW-driven displacement square $\left\langle z_{\text {SAW }}^{2}\right\rangle$ of about $8 \mathrm{pm}^{2}$. We can roughly compare the linear displacement driven by the coherent SAW and by thermal motion by considering the ratio of the square of DSD, i.e., $\eta_{\text {coherent }}=\sqrt{\left\langle z_{\text {SAW }}^{2}\right\rangle} / \sqrt{\left\langle z_{\text {th }}^{2}\right\rangle}$. A larger injection of coherent vibrations translates in a $\eta_{\text {coherent }}$ in a range from 40 to 65 , where the different mechanical modes have been considered. On average, our coherent control of the mechanical motion is about 50 times stronger than the thermal noise, granting a strong and reliable SAW-based operation even when working at room temperature. Of course, our rough estimate suffers from multiple limitations, starting from the assumptions leading to Eq. (1), as having a single mechanical mode with low damping; more accurate evaluations are performed in dedicated sets of samples.

Although a strong vibration amplitude can be reached, the spatial shape of the nanostring displacement of Figs. 6(c) and 6(d) do not show a nice harmonic form at odds with the weaker substrate signals reported in Fig. 6(c). This is due to the fact that the nanostring geometry is not optimized to work around $1 \mathrm{GHz}$, resulting in excitation of high-order modes, further complicated by the presence of the hanging frame, which, as we have seen, plays an important role regarding the whole system excitation. Some insights on the mechanical excited modes can be obtained by considering line scans crossing the nanostring along its width. Such a measurement is reported in Fig. 7(a), along with a visual help of the scan lines superimposed on the optical microscope image. Even if a straight comparison between the measurement obtained and device geometry is not easily done due to the rough resolution of the stage movement, it is possible to observe that few scans have displacement antinodes at the nanobeam edges and nodes at the nanobeam center. Clearly, this is not an optimized configuration when working with a laser spot size of the same order of magnitude of the nanostring lateral width $(1.5 \mu \mathrm{m})$. Nevertheless, the experimental data can be tentatively compared with finite-element-method simulations (COMSOL Multiphysics) of the nanostring plus hanging frame geometry. 
(a)
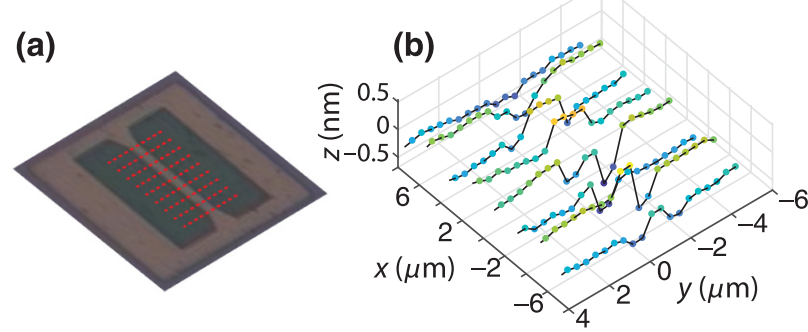

(c)

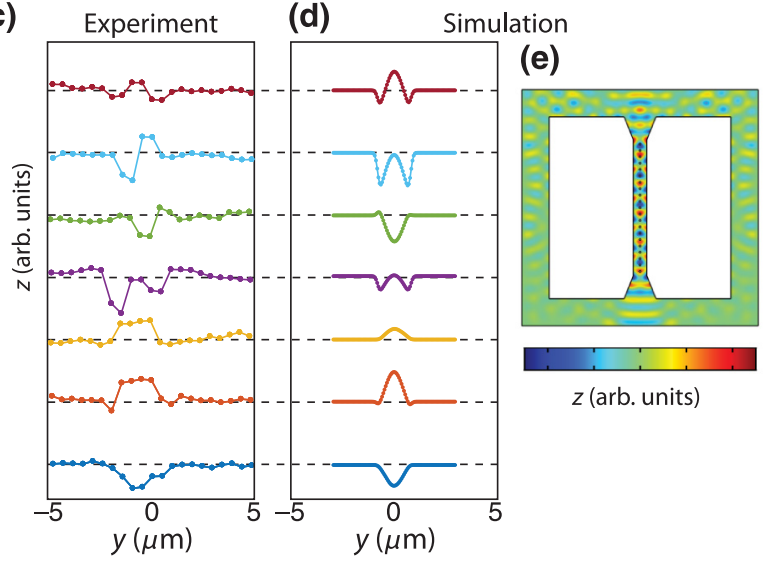

FIG. 7. (a) Profile of the scan lines on top of the nanostring for the measurement reported in (b). Waterfall plot of experimental (c) and simulated displacement (d) obtained from a time-domain simulation. (e) Full map of the simulation.

Nanocrystalline Si is simulated using a 157-GPa Young's modulus and 0.22 Poisson's ratio, which are compatible with values reported in the literature [34]. In order to reproduce our hybrid standing- and traveling-wave system, we run time-domain simulations, in which a $1-\mathrm{GHz}$ mechanical source at one of the nanostring edges is slowly turned on; after a transient phase, we can then estimate the displacement field propagating into the nanostring. A qualitative comparison of the experimental data and simulations can be done by inspecting the waterfall plot of (b) and (c) in Fig. 7. Here the phase of the measurement and simulation time are chosen to optimize a qualitative-data comparison. Moreover, the simulation data is postprocessed by considering a convolution with a Gaussian function of 300 $\mathrm{nm}$ width, roughly representing the effect of a finite laserbeam profile. Even if the spatial resolution required for a more quantitative comparison is beyond our stage movement capabilities, we can find a good qualitative agreement in most of the reported line scans, suggesting that we are experimentally observing the simulated mode.

\section{CONCLUSIONS}

In this paper we show a GHz electromechanical system where a Si nanostring is excited through SAW generated in a piezoelectric AlN layer embedded in the chip. Our hybrid system allows the use of high-frequency SAW with silicon, which has good characteristics for electronics and photonics technologies. Our approach mixes together the expertise of fabrication of Si-based devices and the versatility of SAW, which are already making some of the best high-frequency filters overall and are slowly entering the world of quantum applications [35]. The AlN/Si platform is completely silicon compatible and competes with other monolithic and hybrid approaches showing an external mechanical excitation efficiency of $519 \mathrm{pm} / \mathrm{V}$, using optimized IDT with a simple not-optimized Si nanostring, making it feasible to reach very strong mechanical drives with applied biases of just few volts. This makes the system appealing for low power, high-frequency optical modulation and rf filtering. Furthermore, the device characterization has been performed in ambient conditions (room temperature and atmospheric pressure), showing the robustness of this technology against thermal noise and pressure-induced damping. Future device realizations will see the use of proper optomechanicals crystal replacing the nanostring, with the added value of increased sensitivity to mechanical displacement as well as the possibility to coherently drive the optomechanical crystal for highly efficient, unidirectional wavelength conversion from microwaves to optical regime.

\section{ACKNOWLEDGMENTS}

This work is supported by the FET-Open PHENOMEN project (GA 713450). D.N.U. gratefully acknowledges funding from a Ramn y Cajal postdoctoral fellowship (RYC-2014-15392).

[1] Y. T. Yang, C. Callegari, X. L. Feng, K. L. Ekinci, and M. L. Roukes, Zeptogram-scale nanomechanical mass sensing, Nano Lett. 6, 583 (2006).

[2] J. Chaste, A. Eichler, J. Moser, G. Ceballos, R. Rurali, and A. Bachtold, A nanomechanical mass sensor with yoctogram resolution, Nat. Nanotechnol. 7, 301 (2012).

[3] J. Moser, J. Gttinger, A. Eichler, M. J. Esplandiu, D. E. Liu, M. I. Dykman, and A. Bachtold, Ultrasensitive force detection with a nanotube mechanical resonator, Nat. Nanotechnol. 8, 493 (2013).

[4] A. G. Krause, M. Winger, T. D. Blasius, Q. Lin, and O. Painter, A high-resolution microchip optomechanical accelerometer, Nat. Photonics 6, 768 (2012).

[5] M. Winger, T. D. Blasius, T. P. M. Alegre, A. H. SafaviNaeini, S. Meenehan, J. Cohen, S. Stobbe, and O. Painter, A chip-scale integrated cavity-electro-optomechanics platform, Opt. Express 19, 24905 (2011).

[6] A. Pitanti, J. M. Fink, A. H. Safavi-Naeini, J. T. Hill, C. U. Lei, A. Tredicucci, and O. Painter, Strong opto-electromechanical coupling in a silicon photonic crystal cavity, Opt. Express 23, 3196 (2015).

[7] K. C. Balram, M. I. Davano, J. D. Song, and K. Srinivasan, Coherent coupling between radiofrequency, optical 
and acoustic waves in piezo-optomechanical circuits, Nat. Photonics 10, 346 (2016).

[8] S. Zanotto, A. Tredicucci, D. Navarro-Urrios, M. Cecchini, G. Biasiol, D. Mencarelli, L. Pierantoni, and A. Pitanti, Optomechanics of chiral dielectric metasurfaces, Adv. Opt. Mater. 8, 1901507 (2020).

[9] M. Aspelmeyer, T. J. Kippenberg, and F. Marquardt, Cavity optomechanics, Rev. Mod. Phys. 86, 1391 (2014).

[10] J. Chan, T. P. M. Alegre, A. H. Safavi-Naeini, J. T. Hill, A. Krause, S. Grblacher, M. Aspelmeyer, and O. Painter, Laser cooling of a nanomechanical oscillator into its quantum ground state, Nature 478, 89 (2011).

[11] J. D. Teufel, T. Donner, D. Li, J. W. Harlow, M. S. Allman, K. Cicak, A. J. Sirois, J. D. Whittaker, K. W. Lehnert, and R. W. Simmonds, Sideband cooling of micromechanical motion to the quantum ground state, Nature 475, 359 (2011).

[12] R. W. Andrews, R. W. Peterson, T. P. Purdy, K. Cicak, R. W. Simmonds, C. A. Regal, and K. W. Lehnert, Bidirectional and efficient conversion between microwave and optical light, Nat. Phys. 10, 321 (2014).

[13] A. Vainsencher, K. J. Satzinger, G. A. Peairs, and A. N. Cleland, Bi-directional conversion between microwave and optical frequencies in a piezoelectric optomechanical device, Appl. Phys. Lett. 109, 033107 (2020).

[14] G. Arnold, M. Wulf, S. Barzanjeh, E. S. Redchenko, A. Rueda, W. J. Hease, F. Hassani, and J. M. Fink, Converting microwave and telecom photons with a silicon photonic nanomechanical interface, arXiv:2002.11628 [quant-ph] (2020).

[15] J. T. Hill, A. H. Safavi-Naeini, J. Chan, and O. Painter, Coherent optical wavelength conversion via cavity optomechanics, Nat. Commun. 3, 1196 (2012).

[16] J. Fink, M. Kalaee, R. Norte, A. Pitanti, and O. Painter, Efficient microwave frequency conversion mediated by a photonics compatible silicon nitride nanobeam oscillator, Quantum Sci. Technol. 5, 034011 (2020).

[17] M. V. Gustafsson, T. Aref, A. F. Kockum, M. K. Ekstrm, G. Johansson, and P. Delsing, Propagating phonons coupled to an artificial atom, Science 346, 207 (2014).

[18] K. J. Satzinger, Y. P. Zhong, H.-S. Chang, G. A. Peairs, A. Bienfait, M.-H. Chou, A. Y. Cleland, C. R. Conner, É. Dumur, J. Grebel, I. Gutierrez, B. H. November, R. G. Povey, S. J. Whiteley, D. D. Awschalom, D. I. Schuster, and A. N. Cleland, Quantum control of surface acoustic-wave phonons, Nature 563, 661 (2018).

[19] D. Hutchins, in Physical Acoustics (Academic Press, New York, 1988), p. 21.

[20] D. Munk, M. Katzman, M. Hen, M. Priel, M. Feldberg, T. Sharabani, S. Levy, A. Bergman, and A. Zadok, Surface acoustic wave photonic devices in silicon on insulator, Nat. Commun. 10, 4214 (2019).
[21] M. Wu, E. Zeuthen, K. C. Balram, and K. Srinivasan, Microwave-To-Optical Transduction Using a Mechanical Supermode for Coupling Piezoelectric and Optomechanical Resonators, Phys. Rev. Appl. 13, 014027 (2020).

[22] F. Beil, A. Wixforth, and R. Blick, Investigation of nanoelectromechanical-systems using surface acoustic waves, Phys. E: Low-Dimensional Syst. Nanostruct. 13, 473 (2002).

[23] M. Forsch, R. Stockill, A. Wallucks, I. Marinkovi, C. Grtner, R. A. Norte, F. van Otten, A. Fiore, K. Srinivasan, and S. Grblacher, Microwave-to-optics conversion using a mechanical oscillator in its quantum ground state, Nat. Phys. 16, 69 (2020).

[24] D. Hatanaka and H. Yamaguchi, Real-Space Characterization of Cavity-Coupled Waveguide Systems in Hypersonic Phononic Crystals, Phys. Rev. Appl. 13, 024005 (2020).

[25] L. Fan, X. Sun, C. Xiong, C. Schuck, and H. X. Tang, Aluminum nitride piezo-acousto-photonic crystal nanocavity with high quality factors, Appl. Phys. Lett. 102, 153507 (2013).

[26] H. Liang, R. Luo, Y. He, H. Jiang, and Q. Lin, High-quality lithium niobate photonic crystal nanocavities, Optica 4, 1251 (2017).

[27] B. Dong, X. Luo, S. Zhu, M. Li, D. Hasan, L. Zhang, S. J. Chua, J. Wei, Y. Chang, G.-Q. Lo, K. W. Ang, D.L. Kwong, and C. Lee, Aluminum nitride on insulator (alnoi) platform for mid-infrared photonics, Opt. Lett. 44, 73 (2019).

[28] P. Boucher, S. Rauwerdink, A. Tahraoui, C. Wenger, Y. Yamamoto, and P. V. Santos, Ring waveguides for gigahertz acoustic waves on silicon, Appl. Phys. Lett. 105, 161904 (2014)

[29] G. Harbeke, Growth and physical properties of lpcvd polycrystalline silicon films, J. Electrochem. Soc. 131, 675 (1984).

[30] D. Navarro-Urrios, N. E. Capuj, J. Maire, M. Colombano, J. Jaramillo-Fernandez, E. Chavez-Angel, L. L. Martin, L. Mercad, A. Griol, A. Martnez, C. M. Sotomayor-Torres, and J. Ahopelto, Nanocrystalline silicon optomechanical cavities, Opt. Express 26, 9829 (2018).

[31] D. Navarro-Urrios, N. E. Capuj, J. Gomis-Bresco, F. Alzina, A. Pitanti, A. Griol, A. Martínez, and C. M. S. Torres, A self-stabilized coherent phonon source driven by optical forces, Sci. Rep. 5, 15733 (2015).

[32] M. M. de Lima, F. Alsina, W. Seidel, and P. V. Santos, Focusing of surface-acoustic-wave fields on (100) GaAs surfaces, J. Appl. Phys. 94, 7848 (2003).

[33] D. Morgan, Surface Acoustic Wave Filters (Academic Press, London, 2007).

[34] X. Lu, L. Cui, and M. Ren, Young's modulus of nanopolycrystalline silicon film, Proc. Eng. 29, 4025 (2012).

[35] P. Delsing et al., The 2019 surface acoustic waves roadmap, J. Phys. D: Appl. Phys. 52, 353001 (2019). 